\title{
Temporal lobe epilepsy
}

\section{Hippocampal pathology modulates connectome topology and controllability}

Boris C. Bernhardt, PhD, Fatemeh Fadaie, MSc, Min Liu, PhD, Benoit Caldairou, PhD, Shi Gu, PhD, Elizabeth Jefferies, PhD, Jonathan Smallwood, PhD, Danielle S. Bassett, PhD, Andrea Bernasconi, MD, and Neda Bernasconi, MD, PhD

Neurology ${ }^{\circledR}$ 2019;92:e2209-e2220. doi:10.1212/WNL.0000000000007447

\section{Abstract}

\section{Objective}

To assess whether hippocampal sclerosis (HS) severity is mirrored at the level of large-scale networks.

\section{Methods}

We studied preoperative high-resolution anatomical and diffusion-weighted MRI of 44 temporal lobe epilepsy (TLE) patients with histopathologic diagnosis of $\mathrm{HS}(\mathrm{n}=25$; TLE-HS) and isolated gliosis ( $n=19$; TLE-G) and 25 healthy controls. Hippocampal measurements included surface-based subfield mapping of atrophy and T2 hyperintensity indexing cell loss and gliosis, respectively. Whole-brain connectomes were generated via diffusion tractography and examined using graph theory along with a novel network control theory paradigm that simulates functional dynamics from structural network data.

\section{Results}

Compared to controls, we observed markedly increased path length and decreased clustering in TLE-HS compared to controls, indicating lower global and local network efficiency, while TLEG showed only subtle alterations. Similarly, network controllability was lower in TLE-HS only, suggesting limited range of functional dynamics. Hippocampal imaging markers were positively associated with macroscale network alterations, particularly in ipsilateral CA1-3. Systematic assessment across several networks revealed maximal changes in the hippocampal circuity. Findings were consistent when correcting for cortical thickness, suggesting independence from gray matter atrophy.

\section{Conclusions}

Severe HS is associated with marked remodeling of connectome topology and structurally governed functional dynamics in TLE, as opposed to isolated gliosis, which has negligible effects. Cell loss, particularly in CA1-3, may exert a cascading effect on brain-wide connectomes, underlining coupled disease processes across multiple scales.

\author{
Correspondence \\ Dr. N. Bernasconi \\ neda@bic.mni.mcgill.ca
}




\section{Glossary}

AAL = automated anatomical labeling; FA = fractional anisotropy; FDR = false discovery rate; FOV = field of view; HS = hippocampal sclerosis; MPRAGE = magnetization-prepared rapid gradient echo; ROI = region of interest; TE = echo time; TI = inversion time; TLE = temporal lobe epilepsy; TLE-G = temporal lobe epilepsy with gliosis; TLE-HS = temporal lobe epilepsy with hippocampal sclerosis; TR = repetition time.

Temporal lobe epilepsy (TLE) is the most common drugresistant epilepsy in adults, with hippocampal sclerosis (HS) at its core. ${ }^{1}$ MRI has provided important insights in the understanding of histopathologic variability across patients, with measures of atrophy and T2 signal providing increasingly accurate surrogates of cell loss and gliosis. ${ }^{2-4}$ MRI studies have also been instrumental in revealing whole-brain structural compromise of multiple gray matter regions ${ }^{5,6}$ and white matter tracts. ${ }^{7-9}$ Together with graph theoretical investigations showing perturbed topology of brain networks, ${ }^{10-12}$ findings collectively indicate that TLE affects multiple brain systems. ${ }^{13-15}$

Despite the widely accepted conceptualization of TLE as a network disorder, surprisingly little work has addressed the interplay between hippocampal and connectome-level disease effects. Analyses of structural connectomes based on diffusion MRI have mainly focused on preoperative markers, ${ }^{10}$ disregarding the severity of hippocampal pathology. Even at the level of imaging, no study has systematically related hippocampal in vivo markers to white matter connectome features. Finally, and despite the general consensus that structural networks largely constrain functional connectivity, ${ }^{16}$ it remains unknown whether TLE-related structural connectome alterations affect functional dynamics. Methodologic advances in network science have extended conventional graph theoretical descriptive analysis of topology ${ }^{17}$ by approaches such as network control theory that address structure-function links mechanistically, specifically in predicting how the brain moves between functional states drawn from white matter network organization. By simulating consequences of structural connectome reorganization on brain dynamics, this novel framework lends a novel perspective to examine structurally governed macroscale dysfunction in TLE. $^{18,19}$

To address the interplay between hippocampal and wholebrain anomalies in TLE, we combined hippocampal subfield imaging with diffusion MRI connectomics in patients with histologically confirmed HS and healthy controls. Given the dense connectivity of the hippocampus, particularly to default and temporo-limbic networks, ${ }^{20}$ we hypothesized that interpatient variations in HS severity would be reflected in the degree of white matter connectome reorganization. We parametrized network topology using graph theory and simulated structurally governed brain dysfunction using network control theory. ${ }^{18,19}$ To assess specificity, we compared effects of networks centered on the hippocampus to other intrinsic networks and repeated analyses after correcting for effects of cortical atrophy. Findings were validated across parcellation schemes and alternate topologic measures.

\section{Methods}

\section{Participants}

From a database of patients referred to our hospital for the investigation of drug-resistant TLE, we selected a consecutive cohort of 44 TLE patients ( 17 male; $18-53$ years, mean \pm SD age $33 \pm 9$ years) based on the following inclusion criteria: (1) patients underwent surgery; (2) the resected specimen was suitable for histologic analysis ${ }^{21}$; and (3) patients had a research-dedicated high-resolution 3T MRI prior to surgery that included anatomical and diffusion MRI. Demographic and clinical data were obtained through interviews with patients and their relatives. TLE diagnosis and lateralization of the seizure focus into left TLE $(\mathrm{n}=20)$ and right TLE $(\mathrm{n}=$ 24) were determined by a comprehensive evaluation including detailed history, neurologic examination, review of medical records, video-EEG recordings, and clinical MRI evaluation in all patients. Notably, no patient had a mass lesion (malformations of cortical development, tumor, vascular malformations) or a history of traumatic brain injury or encephalitis.

Mean follow-up time after surgery was $80 \pm 20$ months (36-120 months). We determined postsurgical seizure outcome according to Engel modified classification. ${ }^{22}$ Thirtythree $(75 \%)$ patients had a Class I outcome (i.e., free of disabling seizures). Residual seizures were noted in the remaining 11 cases ( 6 Class II, 4 Class III, 1 Class IV). Based on histopathologic criteria, ${ }^{21} 25$ patients showed hippocampal cell loss and gliosis (TLE-HS), including 15 with marked cell loss in both CA1 and CA3 (type 1), 7 with CA1predominant (type 2), and 3 with CA4-predominant cell loss (type 3). Nineteen patients presented with isolated gliosis (TLE-G).

Comparing demographic and clinical measures (using Student $t$ tests and Fisher exact tests, respectively), we noted no significant differences in age (TLE-HS: $32 \pm 9$ years; TLE-G: $34 \pm 10$ years; $p>0.4$ ), male/female distribution (TLE-HS: 12/13; TLE-G: $5 / 14 ; p>0.20$ ), left/right seizure foci (TLEHS: $12 / 13$; TLE-G: $8 / 11 ; p>0.4$ ), and duration of epilepsy $(19 \pm 11 / 15 \pm 10$ years; $p>0.2)$. While likelihood of postoperative seizure freedom was somewhat higher in TLE-HS than in TLE-G, the difference was not significant (Class I vs 
II-IV in TLE-HS: 21/4; TLE-G: $12 / 7$; $p=0.16$ ). Similarly, patients with TLE-HS had a tendency for an earlier disease onset ( $13 \pm 10$ vs $18 \pm 12$ years; $p=0.09)$ and a higher proportion of patients experiencing generalized tonic-clonic seizures (TLE-HS: 19/6 vs TLE-G: 8/11; $p<0.04$ ). Notably, all analyses were repeated after controlling for age, sex, duration of epilepsy, generalized tonic-clonic seizures, and age at onset to verify the robustness of network differences between TLE-HS vs TLE-G against subthreshold differences in these variables. The proportion of patients with childhood febrile convulsions was higher in TLE-HS (TLE-HS: $14 / 11$ vs TLEG: $2 / 17 ; p<0.005$ ), in keeping with prior pathologic ${ }^{1}$ and imaging findings. ${ }^{23}$ The control group consisted of 25 ageand sex-matched healthy individuals ( 12 male; $21-53$ years; mean \pm SD age $32 \pm 8$ years). See the table for sociodemographic and clinical information on the included study cohorts.

\section{Standard protocol approvals, registrations, and patient consents}

The Ethics Committee of the Montreal Neurological Institute and Hospital approved the study and written informed consent to undergo research-dedicated MRI investigations was obtained from all participants.

\section{MRI acquisition}

MRI in patients and controls were acquired on a 3T Siemens (Erlangen, Germany) TimTrio scanner using a 32-channel head coil. For hippocampal subfield analysis, we acquired submillimetric 3D magnetization-prepared rapid gradient echo (MPRAGE) T1-weighted MRI (repetition time [TR] $3,000 \mathrm{~ms}$, echo time [TE] $4.32 \mathrm{~ms}$, inversion time [TI] 1,500 ms, flip angle $7^{\circ}$, matrix $336 \times 384$, field of view [FOV] $201 \times$ $229 \mathrm{~mm}^{2}, 240$ axial slices with $0.6 \mathrm{~mm}$ thickness, resulting in $0.6 \times 0.6 \times 0.6 \mathrm{~mm}^{3}$ voxels); to increase signal-to-noise ratio, 2 acquisitions were collected, coregistered, and averaged. T2weighted MRI was obtained using a 2D turbo spin-echo sequence (TR $10,810 \mathrm{~ms}, \mathrm{TE} 81 \mathrm{~ms}$, flip angle $119^{\circ}$, matrix size $512 \times 512$, FOV $203 \times 203 \mathrm{~mm}^{2}, 60$ coronal slices titled perpendicular to the hippocampal long axis, thickness $2.0 \mathrm{~mm}$, resulting in $0.4 \times 0.4 \times 2.0 \mathrm{~mm}^{3}$ voxels). Whole-brain cortical surfaces were derived from millimetric 3D MPRAGE T1-weighted MRI (TR 2,300 ms, TE 2.98 ms, TI 900 ms, flip angle $9^{\circ}$, matrix $256 \times 256$, FOV $256 \times 256 \mathrm{~mm}^{2}$, 176 sagittal slices with $1 \mathrm{~mm}$ thickness, resulting in $1 \times 1 \times 1 \mathrm{~mm}^{3}$ voxels). Finally, connectivity matrices were generated from a twicerefocused $2 \mathrm{D}$ echoplanar imaging diffusion MRI sequence (TR 8,400 ms, TE $90 \mathrm{~ms}$, matrix size $128 \times 128$, FOV $256 \times$ $256 \mathrm{~mm}^{2}, 63$ transverse slices with $2.0 \mathrm{~mm}$ thickness, resulting in $2.0 \times 2.0 \times 2.0 \mathrm{~mm}^{3}$ voxels; 64 diffusion directions with $\mathrm{b}=$ 1,000 seconds $/ \mathrm{mm}^{2}$ along with $1 \mathrm{~B}_{0}$ image).

\section{Network analysis}

\section{T1 preprocessing and brain parcellation}

T1-weighted images underwent intensity nonuniformity correction and were linearly registered to Montreal Neurological Institute 152 template space. As in previous work, ${ }^{24,25}$ we extracted surfaces of the cortical mantle using automated procedures. Cortical surfaces were aligned with respect to sulco-gyral folding, improving interindividual correspondence in measurement points (henceforth, vertices). We nonlinearly registered each T1-weighted image to Colin27 single-subject template. ${ }^{26}$ The resulting deformation field was used to map the automated anatomical labeling (AAL) atlas ${ }^{27}$ to T1weighted MRI, thereby parcellating the brain into 90 regions of interest (ROIs) (78 cortical, 12 subcortical; henceforth $\mathrm{AAL}_{\mathrm{LR}}$ ). We also generated a high-resolution parcellation, by subdividing each parcel into smaller patches with similar surface areas constrained by cortical sulcation, resulting in 294 cortical ROIs that were added to the original 12 subcortical labels (for a total of 306 areas, henceforth, $\mathrm{AAL}_{\mathrm{HR}}$ ).

\section{Connectome construction}

Diffusion MRI data underwent correction for geometric distortions, eddy currents, and head motion. Patients and controls did not differ with respect to head motion measures (translation: $|t|<0.7, p>0.2$; rotation: $|t|<0.52, p>0.4$ ). We estimated the shape of the diffusion tensor at each voxel and derived fractional anisotropy (FA) using trackvis (trackvis. org/). We mapped cortical surfaces and AAL parcellations from T1-weighted MRI to native diffusion space, using a nonlinear registration between the white matter partial volume classification derived from preprocessed T1-weighted images $^{28}$ and the FA skeleton. For both parcellations (i.e., $\mathrm{AAL}_{\mathrm{LR}}$ and $\mathrm{AAL}_{\mathrm{HR}}$ ), we propagated labels into the

Table Demographic and clinical information of the temporal lobe epilepsy cohorts and healthy controls

\begin{tabular}{|c|c|c|c|c|c|c|c|c|}
\hline Group & Male & $\begin{array}{l}\text { Age, } y \\
\text { mean } \pm \text { SD } \\
\text { (range) }\end{array}$ & L/R & $\begin{array}{l}\text { Onset, } y \text {, } \\
\text { mean } \pm \text { SD } \\
\text { (range) }\end{array}$ & $\begin{array}{l}\text { Duration, y, } \\
\text { mean } \pm \text { SD } \\
\text { (range) }\end{array}$ & GCTS, n (\%) & FC, n (\%) & Engel I, n (\%) \\
\hline Controls $(n=25)$ & 12 & $32 \pm 8(21-53)$ & - & - & - & - & - & - \\
\hline TLE-HS $(n=25)$ & 13 & $32 \pm 9(18-53)$ & $12 / 13$ & $13 \pm 8(1-27)$ & $19 \pm 11(1-45)$ & $19(76)$ & $14(56)$ & $21(84)$ \\
\hline TLE-G $(n=19)$ & 14 & $34 \pm 10(19-53)$ & $8 / 11$ & $18 \pm 12(3-51)$ & $15 \pm 10(2-35)$ & $8(42)$ & $2(11)$ & $12(63)$ \\
\hline
\end{tabular}

Abbreviations: Engel I = seizure-free postsurgical outcome according to Engel classification; FC = history of childhood febrile convulsions; GTCS = history of generalized tonic-clonic seizures; L/R = left/right sided EEG seizure focus; TLE-G = temporal lobe epilepsy with gliosis; TLE-HS = temporal lobe epilepsy with hippocampal sclerosis. 
superficial white matter $1 \mathrm{~mm}$ below the gray-white matter boundary using our recent approach, ${ }^{29}$ where cortical vertices are moved towards the ventricles along a Laplacian field. We generated whole-brain structural connectomes for both spatial scales using streamline tractography based on fiber assignment by continuous tracking (FACT), an algorithm that accounts for multiple diffusion directions in a voxel. ${ }^{30}$ The propagation is constrained within the white matter and halted when reaching $1 \mathrm{~mm}$ below the white-gray matter interface or when streamlines exceed a curvature threshold of 60 . Based on these streamlines, we sampled subject-wise average FA for each inter-regional pair and stored the data in the whole-brain connectivity matrix. As connectivity strength was quantified by FA and not numbers of streamlines, we did not control for distance between 2 given ROIs. ${ }^{31}$

\section{Network topology}

Using scripts from the Brain Connectivity Toolbox, ${ }^{32}$ we computed the clustering coefficient and characteristic path length for the whole connectome in controls and patients using standard formulas. Clustering coefficient is a marker of cliquishness and is correlated to local efficiency ${ }^{33}$; here we calculated clustering as the mean clustering coefficient across all nodes. After calculating the shortest path from every node to all other brain regions (or nodes), we computed the characteristic path length as the average of the shortest path length across all nodes. Long paths signify low global efficiency, which was calculated as the average of the inverse shortest path lengths. ${ }^{33}$

\section{Network controllability}

We leveraged a recently developed network control theory framework to calculate node- and network-wide controllability. ${ }^{18,19}$ Inputs to this approach are a structural connectome (as defined in the connectome construction section) and a model for the dynamics of neural processes based on prior work in human systems neuroscience; the latter is based on models linking structural brain networks to resting-state functional signals. ${ }^{16}$ Network control theory, in turn, defines a trajectory of an interconnected system as the temporal path that the system traverses through different states, where a state is defined as the magnitude of neurophysiologic activity across brain regions at a single time point. Trajectories can be understood as dynamic changes in brain-wide simulated functional activation patterns. Average controllability of a node indexes the ability of that node to influence others and is calculated as the average input energy needed to reach all possible states of the system (i.e., the overall magnitude of energy measured across all nodes). ${ }^{17}$ In nonrandom networks, regions with high average controllability generally also have high centrality, and thus serve as hubs playing an important role in maintaining macroscale connectome organization. ${ }^{34}$ Despite this resemblance, controllability metrics translate more directly into the overall functional dynamics than centrality measures. Parcel-wise controllability was sorted into ipsilateral and contralateral to the seizure focus following a $z$ - normalization relative to controls, ${ }^{35}$ thereby accounting for interhemispheric asymmetry of healthy individuals.

\section{Relation to hippocampal imaging measures}

We first segmented hippocampal subfields ${ }^{36}$ using our previously developed patch-based multitemplate algorithm. ${ }^{37}$ Similar to our previous findings, ${ }^{38,39}$ TLE-HS presented with reduced global ipsilateral hippocampal volume compared to controls $(t>7.2, p<0.001)$ that was consistent across all subfields albeit with highest effects in CA1-3 ( $t$ values for subiculum: 5.5, CA1-3: 7.0, CA4-DG: 4.8). No effects were seen contralaterally $(|t|<0.9)$. Similar effects were also seen when comparing TLE-HS to TLE-G ipsilaterally, with more pronounced global $(t=8.1)$ and subfield-level atrophy in the former $(t>5.4)$. We then generated medial sheet representations of these labels, ${ }^{40}$ and parameterized them using spherical harmonic shape descriptors. These surfaces, which run through the core of individual subfields, allow for vertexwise computation of local features with minimal partial volume effects, and guarantee shape-inherent vertex correspondence across individuals. ${ }^{40}$ To assess atrophy, we estimated vertex-wise volumes between the subfield boundary and the medial surface ${ }^{40}$; for a given medial sheet vertex, columnar volume was calculated by multiplying the distance to the corresponding vertex on the outer subfield surface with the mean area of the triangles whose edges include both vertices. We computed normalized T2 signal intensity by dividing the intensity at a given voxel by the mean $\mathrm{T} 2$ signal in the CSF of the anterior horn of the lateral ventricles. ${ }^{40}$ Hippocampal measures in patients were $z$-scored relative to corresponding measures in controls and sorted into ipsilateral and contralateral to the seizure focus.

\section{Statistical analysis}

Statistical analyses were performed using SurfStat toolbox ${ }^{41}$ for MATLAB (R2017b; The Mathworks, Natick, MA).

\section{Network-level group comparisons}

We compared clustering coefficient, path length, and average controllability in TLE-HS vs healthy controls, TLE-G vs healthy controls, and TLE-HS vs TLE-G using linear models.

\section{Correlation analysis between network markers and hippocampal features}

We computed surface-wide multivariate correlations between the overall load of hippocampal MRI anomalies (i.e., volume, T2) and network indices (i.e., clustering coefficient, path length, controllability). In clusters of findings, we illustrated effects separately for volume and T2 via univariate post hoc correlations. To cover the range of pathology beyond histologic categories, correlations were also performed across all patients regardless of pathologic diagnosis.

\section{Control for atrophy}

To assess changes in connectome organization beyond effects of diffuse gray matter atrophy, we repeated the above graph and controllability analyses after statistically correcting for 
mean cortical thickness, measured as the distance between corresponding vertices on the inner and outer cortical surfaces. $^{24,42}$

\section{Multiple comparisons correction}

Findings were corrected using false discovery rate (FDR) procedures. $^{43,44}$

\section{Data availability}

Phenotypic and mean connectome data are available at doi: 10.6084/m9.figshare.7309214.

\section{Results}

\section{Group differences in network topology and controllability}

The main findings are based on the $\mathrm{AAL}_{\mathrm{LR}}$ parcellation. In a separate reproducibility section (see below), we demonstrate consistency when using $\mathrm{AAL}_{\mathrm{HR}}$.

\section{Network topology}

Considering whole-brain graph theoretical measures (figure 1), TLE-HS presented with markedly increased path length and decreased clustering coefficient compared to controls
(FDR-corrected $p$ value, $p_{\mathrm{FDR}}<0.05$; Cohen $d=1.20-1.98$ ), while TLE-G showed smaller effects $(d=0.39-0.88)$ that were only significant for path length after correction for multiple comparisons. Directly contrasting patient groups, we indeed observed lower clustering coefficient and longer paths in TLEHS compared to TLE-G $\left(p_{\text {FDR }}<0.05 ; d=0.76-0.81\right)$.

\section{Network controllability}

In controls (figure 2A), areas with high controllability included centro-parietal midline and superior frontal regions, replicating earlier findings in a separate cohort. ${ }^{17}$ These regions, which also display high centrality values, represent hubs. ${ }^{17}$ In fact, the rank of average controllability strongly correlated with the rank of degree centrality across parcels $(r=$ 0.92, $\left.p<1^{-15}\right)$, an additional indication of deviation from a random network architecture (where such a relationship may not be seen). ${ }^{34}$

Between-group comparisons of whole-brain controllability (figure 2B) showed a similar pattern as the graph theoretical analysis, supporting the notion that structural topology largely constrains functional dynamics. While controllability was decreased in both TLE-HS and TLE-G compared to controls $\left(p_{\mathrm{FDR}}<0.05\right)$, effects were more marked in the

\section{Figure 1 Connectome analysis}

\section{A. Structural connectomes}
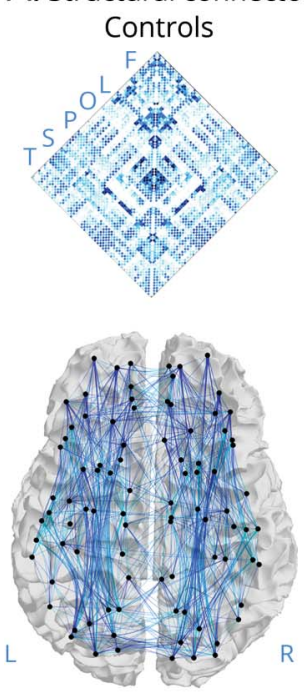
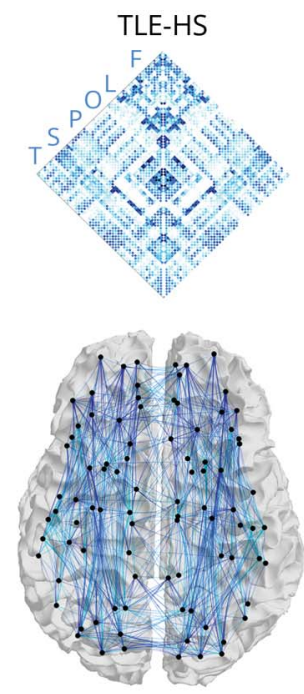
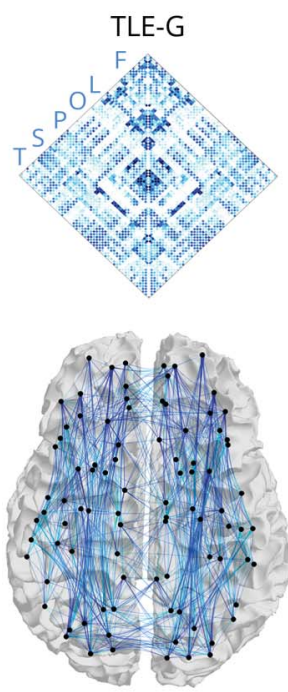

B. Topological parameter analysis
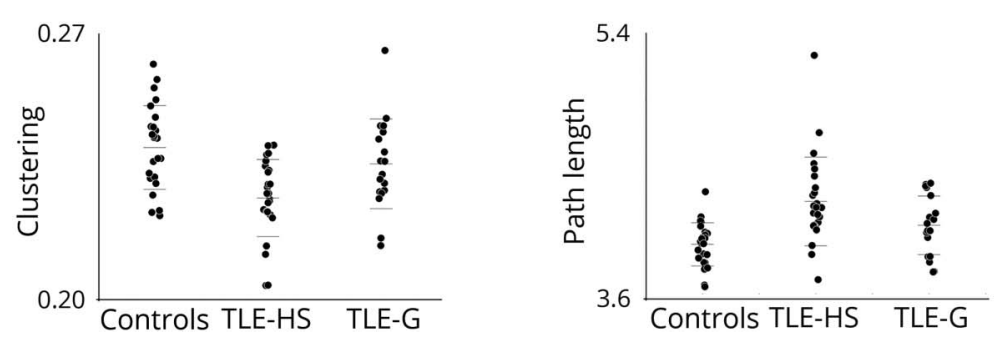

(A) Whole-brain structural connectomes in healthy controls, temporal lobe epilepsy patients with histologic confirmed hippocampal sclerosis (TLE-HS), and temporal lobe epilepsy patients with isolated gliosis (TLE-G). Connectomes were generated using systematic diffusion tractography between all regions, parcellated according to automated anatomical labeling. Letters in the matrix refer to regional groupings of the nodes (i.e., $F=$ frontal, $L=$ limbic, $\mathrm{O}=$ occipital, $\mathrm{P}=$ parietal, $\mathrm{S}=$ subcortical, $\mathrm{T}=$ temporal). (B) Graph theoretical topological measures of clustering coefficient and path length highlighting marked alterations in patients with TLE-HS, while those with TLE-G are only moderately affected compared to controls. 
A. Network controllability in healthy individuals
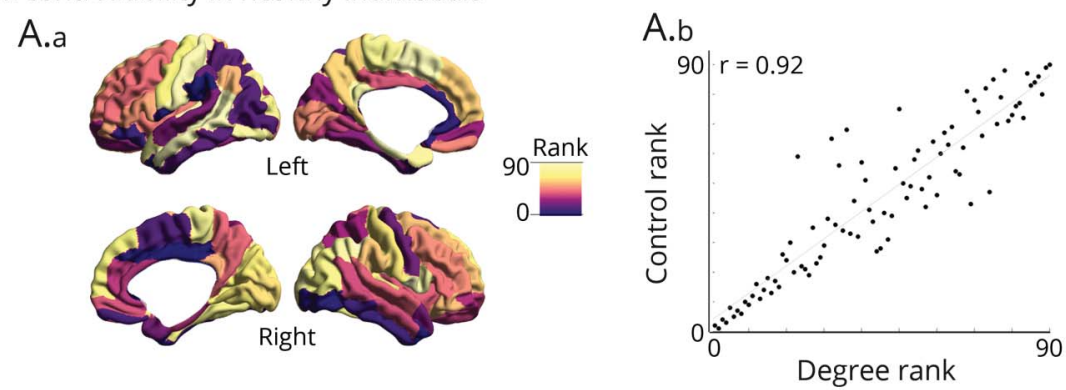

B. Controllability alterations in TLE
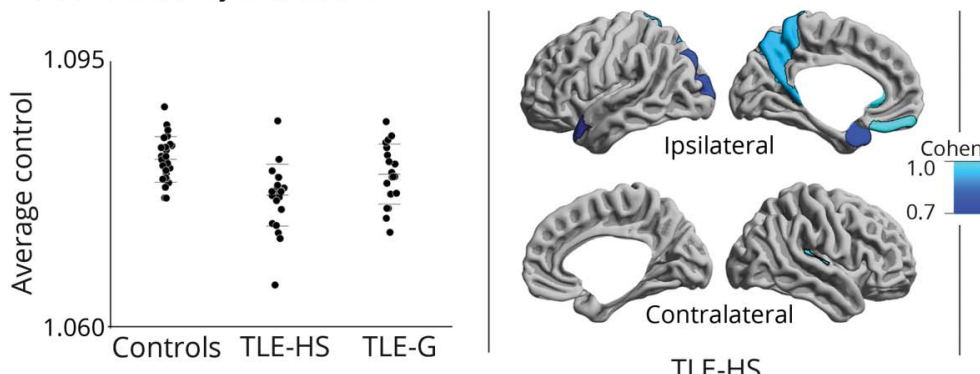

TLE-HS

(A) Controllability mapping in healthy controls. (A.a) Regional controllability ranked across areas. (A.b) Relation between rank of controllability and degree centrality, indicating that more central regions have larger influence on system dynamics. (B) Alterations in controllability in temporal lobe epilepsy with hippocampal sclerosis (TLE-HS) and temporal lobe epilepsy with gliosis (TLE-G) relative to controls. Maps show Cohen $d$ effect size in $p_{\mathrm{FDR}}<0.05$ corrected findings. FDR = false discovery rate.

former (TLE-HS: $d=1.57$, TLE-G: $d=0.64$ ). Directly comparing patient cohorts, TLE-HS was indeed more severely affected than TLE-G $\left(p_{\mathrm{FDR}}<0.05 ; d=0.69\right)$. Regional analysis mapped a specific pattern of alterations in TLE-HS, with lower controllability in ipsilateral anterior temporal and temporopolar, together with medial prefrontal and posterior midline regions, including retrosplenial cortex and precuneus $\left(p_{\mathrm{FDR}}<0.05\right)$. Contralateral decreases were confined to a small region in the posterior insula. Findings in TLE-G, on the other hand, did not survive correction for multiple comparisons.

\section{Specificity of findings with respect to functional networks}

For all group comparisons, we systematically compared topologic and controllability changes across several functional networks. In addition to selecting the network centered on the hippocampus/parahippocampus (based on the tractographic connections in healthy controls), we studied networks centered on somatomotor (pre/postcentral), visual (calcarine/inferior occipital), default (precuneus/medial prefrontal), frontoparietal (superior frontal, inferior parietal), and salience (anterior/ midcingulate, insula). Notably, despite all networks being affected in TLE compared to healthy controls, effects were highest in hippocampal/parahippocampal networks (mean absolute Cohen $d$ in hippocampal/parahippocampal $=0.83$ compared to $0.45-0.75$ in other networks) (figure e- 1 ; doi.org/ 10.5061/dryad.v309h90).

\section{Relation to hippocampal imaging measures}

Surface-based multivariate analyses assessed correlations between whole-brain network markers (i.e., clustering coefficient, path length, and controllability) and the load of hippocampal anomalies, quantified through columnar volume and T2 intensity across TLE patients (figure 3 ). For all network measures, we observed a consistent correlation with hippocampal anomalies across all subfields bilaterally, particularly marked in average controllability $\left(p_{\text {FDR }}<0.1\right)$. The most consistent areas of overlap were, however, in the ipsilateral $\mathrm{CA} 1-3$.

Post hoc univariate correlations in clusters of findings illustrated the association between each network measure and subfield marker. Specifically, patients with more marked hippocampal atrophy (i.e., less columnar volume) showed more severe network alterations (i.e., lower clustering, longer path lengths, lower controllability) compared to those with less marked volume loss. Associations between hippocampal T2 intensity and network markers followed an inverse pattern, with hyperintensity relating to decreased clustering, increased path length, and decreased controllability.

\section{Relation to neocortical morphology}

Surface-based cortical thickness analysis revealed marked atrophy in both TLE-HS and TLE-G in frontocentral, orbitofrontal, as well as mesiotemporal regions when compared to healthy controls (figure 4A). While effects were more diffuse 
A. Multivariate surface-wide
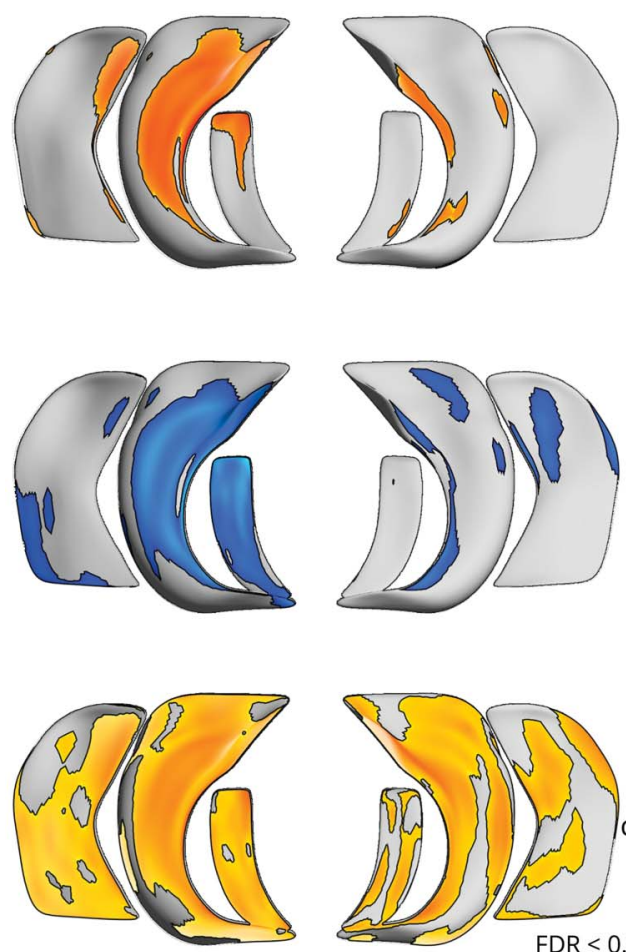

Ipsilateral

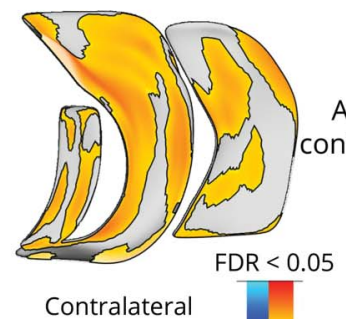

Contralateral
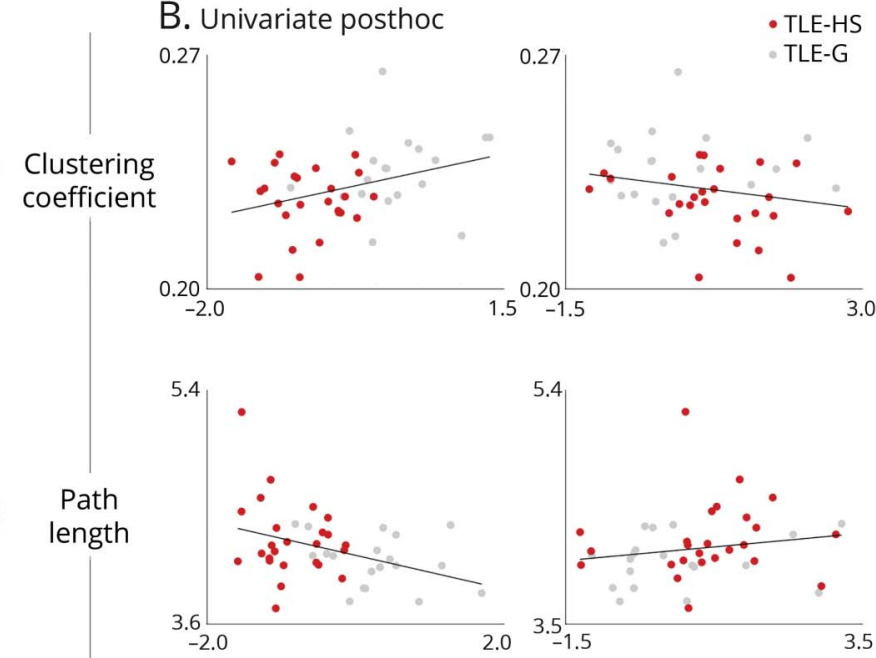

Average controllability
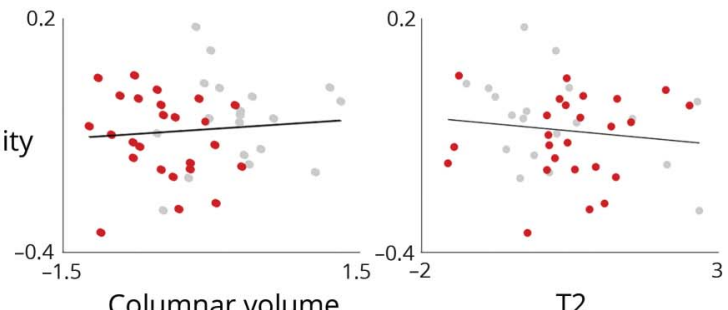

(A) Surface-wide multivariate correlations between network features (clustering coefficient, path length, controllability) and local indices of structural subfield integrity (columnar volume, T2 intensity) across all patients. (B) Post hoc correlation analyses in clusters of findings showing effects for individual subfield markers (red dots: temporal lobe epilepsy with hippocampal sclerosis [TLE-HS]; gray dots: temporal lobe epilepsy with gliosis [TLE-G]). FDR = false discovery rate.

in TLE-HS, differences between patient groups did not survive correction for multiple comparisons. Similar findings were seen at the level of mean cortical thickness, where effect sizes were greater in TLE-HS compared to healthy controls (Cohen $d$ of TLE-HS/TLE-G vs controls: $-0.33 /-0.19$ ), but the direct contrast was not significant (TLE-HS vs TLE-G: $t=$ 0.86, $p>0.2$ ). Notably, group differences in path length, clustering, and controllability remained consistent after correction for cortical thickness $\left(p_{\mathrm{FDR}}<0.05\right)$, suggesting that the between-cohort divergence in connectome organization was robust against effects of global atrophy (figure 4B).

\section{Reproducibility analysis}

\section{Efficiency measures}

To assess reproducibility of topologic findings, we quantified local efficiency (with high values indicating high clustering) and global efficiency (with high values indicating short path length). Similar to clustering and path length, these measures showed marked reductions in TLE-HS $\left(p_{\mathrm{FDR}}<0.05\right.$; Cohen $d>1.65)$ and smaller effects in TLE-G $\left(p_{\mathrm{FDR}}<0.05 ; d>0.68\right)$ (figure e-2; doi.org/10.5061/dryad.v309h90). Contrasting patient groups, structural connectomes in TLE-HS showed decreased global and local efficiency compared to TLE-G $\left(p_{\text {FDR }}<0.05 ; d>0.76\right)$.

\section{Spatial scale}

Repeating topologic and control theory analysis (i.e., clustering, path length, controllability) using the alternative $\mathrm{AAL}_{\mathrm{HR}}$ scheme (figure 5) resulted in similar findings, with decreases in TLE-HS compared to healthy controls for all measures $\left(p_{\mathrm{FDR}}<0.05\right)$, while only trends were found in TLE-G. Moreover, network indices derived from the $\mathrm{AAL}_{\mathrm{HR}}$ parcellation correlated with subfield-level columnar volume and T2 intensity $\left(p_{\mathrm{FDR}}<0.05\right)$, suggesting that the association between large-scale network topology and hippocampal microstructure was consistent across spatial scales.

\section{Clinical and demographic measures}

We repeated network analyses after controlling for age, sex, duration of epilepsy, GTCS, and age at onset to verify whether network differences between TLE-HS vs TLE-G are robust against subthreshold differences in these variables. Indeed, marked alterations in TLE-HS compared to TLE-G were robust even when models additionally included for clinical and sociodemographic characteristics.

\section{Head motion}

We repeated our main analyses after including rotation/ translation measures from the head motion correction of the diffusion MRI data. Again, we could replicate main 


\section{A. Cortical thickness analysis: TLE subgroups vs controls}
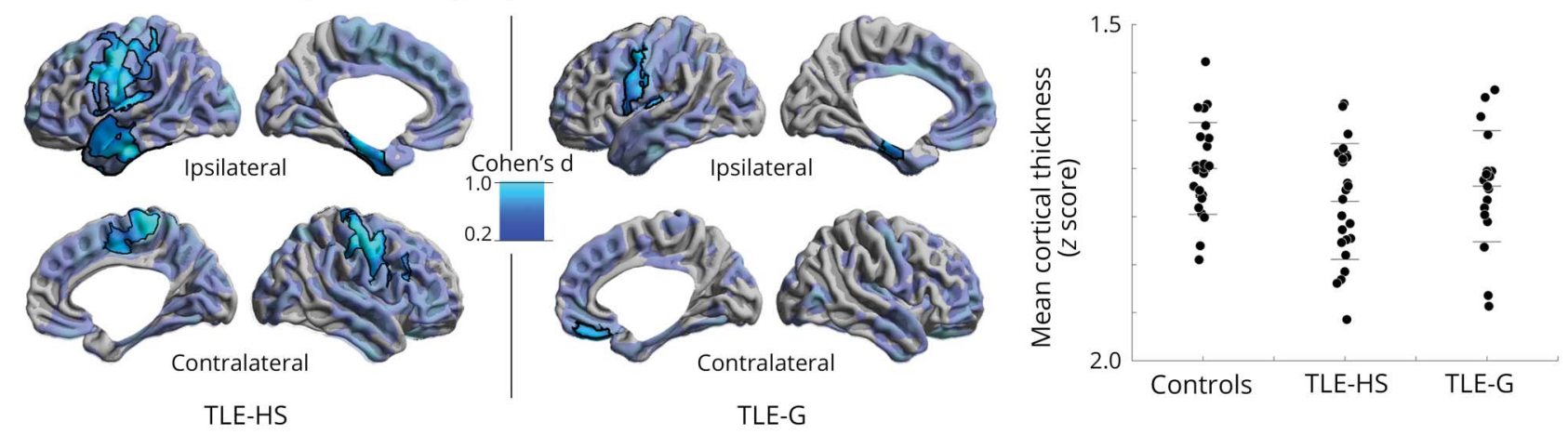

B. Thickness-corrected connectome measures
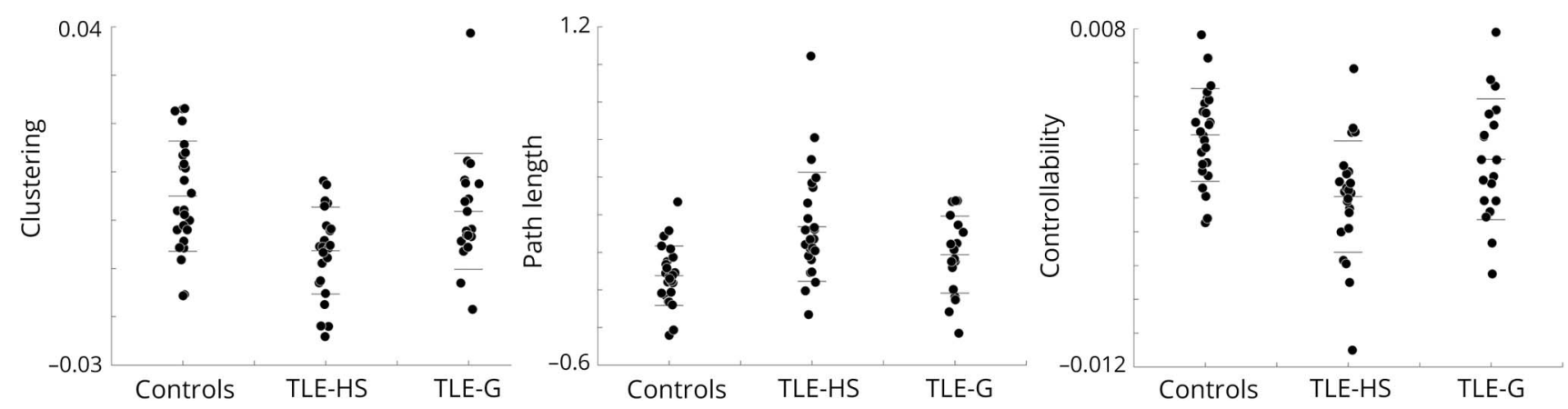

(A) Surface-wide group analysis of cortical thickness between temporal lobe epilepsy with hippocampal sclerosis (TLE-HS) and temporal lobe epilepsy with gliosis (TLE-G) compared to healthy controls. Maps show $p_{\mathrm{FDR}}<0.05$ corrected findings (black outlines) superimposed on Cohen $d$ effect size maps (thresholded at $d>0.2$, semitransparent). Although effect sizes were larger in TLE-HS, there was no significant difference between patient subgroups after the correction for multiple comparisons. Whole-brain mean cortical thickness findings are displayed for the 3 cohorts. (B) Thickness-corrected measures of clustering coefficient, path length, and controllability in the 3 groups, highlighting robustness of the marked effect of TLE-HS on white matter connectome organization.

results showing more marked alterations in TLE-HS than in TLE-G.

\section{Discussion}

This work combined 2 main streams in the neuroimaging of TLE, that is, high-resolution analysis of the hippocampal formation and whole-brain connectomics, including network control theory, a novel framework integrating network structure with models of neural function. Our study provides new evidence that variations in mesiotemporal pathology are main determinants of whole-brain structural network alterations and structurally governed functional dynamics. Indeed, compared to healthy controls, patients with TLE-HS present with marked alterations of network topology and reduced controllability, while TLE-G showed only subtle changes. Specificity for a contribution of hippocampal pathology to interpatient variability in large-scale white matter network reorganization and functional dynamics was suggested by several additional analyses, including a systematic subnetwork assessment revealing maximal changes in the hippocampal/ parahippocampal circuity, high consistency of findings when controlling for overall neocortical thinning, and multivariate correlations between in vivo proxies of hippocampal pathology and network alterations.

A unique aspect of the connectome analysis was a categorization of patients into TLE-HS and TLE-G guided by postoperative histology. We additionally parameterized hippocampal subfield anomalies in vivo through high-resolution and multicontrast measurement of columnar volume and $\mathrm{T} 2$ intensity, ${ }^{40}$ the most widely studied MRI markers of HS. ${ }^{40}$ Correlative studies have shown associations of these markers with histologic grades, both at the whole-hippocampus ${ }^{2,3}$ and subfield-level, ${ }^{4,38}$ supporting their utility as a bridge towards pathology. Notably, multiparameter analysis in a surface-based reference frame that follows hippocampal folding can capture subtle changes that would evade global MRI volumetry, ${ }^{38,45}$ thereby increasing sensitivity in the evaluation of patients with TLE-G. ${ }^{40}$ Moreover, compared to histopathologic analysis, our in vivo framework provides a $3 \mathrm{D}$ and fully quantitative measurement of structural integrity along the entire extent of the hippocampus bilaterally. ${ }^{39,46}$ 
A. AAL subparcellation

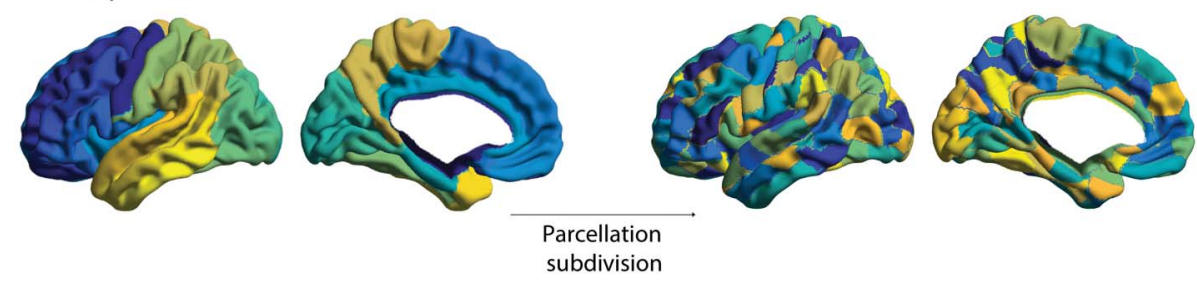

B. High-resolution connectomes in controls and TLE
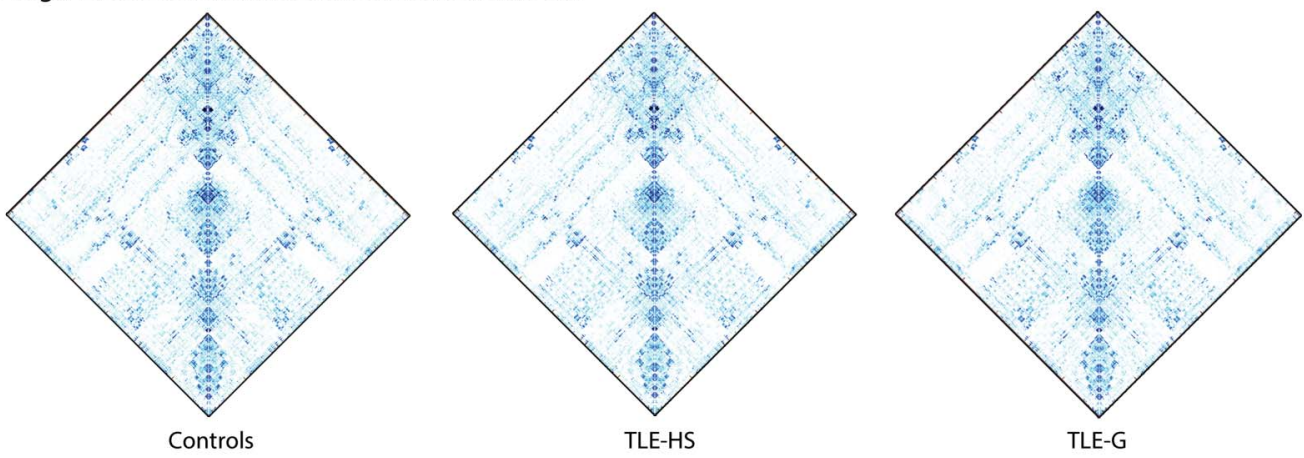

C. Relation to topology and controllability
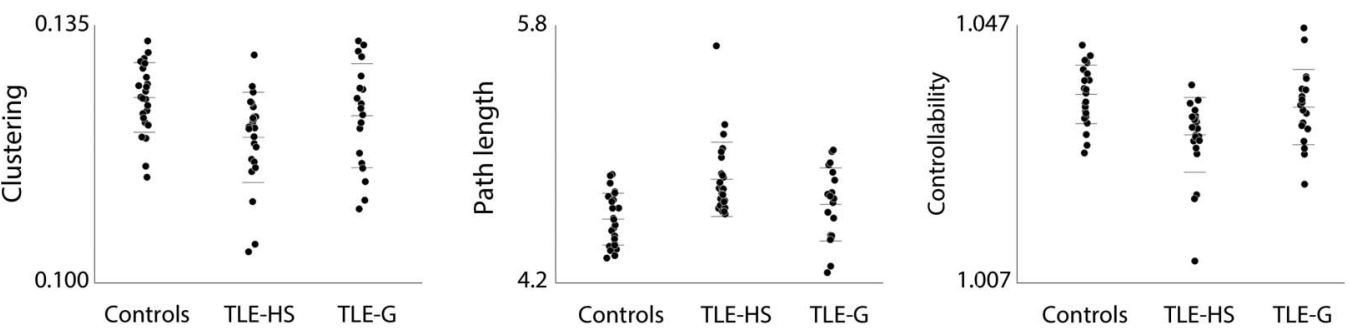

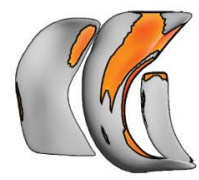

Ipsilateral

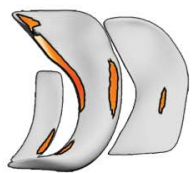

Contralateral
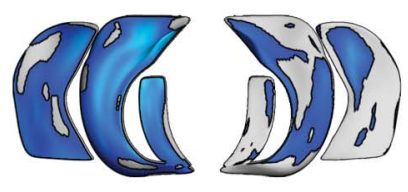

FDR $<0.05$

(A) Subdivision of $A A L_{L R}$ into $A A L_{H R}$. (B) Connectomes in temporal lobe epilepsy (TLE) groups and controls. (C) Differences in clustering coefficient, path length, and controllability across the 3 groups and within-patient multivariate correlations between network measures and columnar volume and $\mathrm{T} 2$ intensity. $\mathrm{AAL}=$ automated anatomical labeling; FDR = false discovery rate; TLE-G = temporal lobe epilepsy with gliosis; TLE-HS = temporal lobe epilepsy with hippocampal sclerosis.

Connectome analysis was based on diffusion tractography allowing for the generation of subject-specific networks. Evaluations at different spatial scales showed increased path length in both TLE-G and TLE-HS, albeit with more marked effects in the latter. These findings, indicative of reduced global efficiency, likely reflect topology-level effects of previously reported diffusion anomalies in multiple long-range tracts in TLE. ${ }^{4748}$ With regards to clustering, a measure of local efficiency, we could also observe reductions in patients, which were again more marked in TLE-HS. Given the relation between clustering and network segregation, these results suggest reduced connectivity, particularly within temporolimbic networks heavily connected to the hippocampus. A stronger extent of white matter anomalies in the proximity of the mesiotemporal lobe is in line with prior network neuroscience studies, ${ }^{49}$ including tract-based assessments showing a tapering effect of diffusion metrics with increasing distance from the focus. ${ }^{47,48}$ As our connectivity matrices were weighted by FA, reductions could theoretically relate to increased fiber crossing in affected networks; however, given previous histopathologic evidence for associations of FA with axonal membrane circumference, myelin fraction, and axonal 
packing, our findings more likely relate to perturbations in tract microstructure. ${ }^{50}$

To address the functional implications of structural connectome reorganization, we harnessed a network control theory paradigm. ${ }^{17}$ Compared to graph theory's metrics describing the static organization of networks, control theory models neural dynamics upon network structure, allowing for mechanistic predictions on the influence of individual regions on network-wide function. Comparisons between patients and healthy controls showed severe controllability reductions in TLE-HS and only subtle effects in TLE-G, suggesting that the structural makeup in the former may have a limiting effect on the repertoire of whole-brain functional dynamics. Findings were complemented with region-specific analysis; in our controls, this approach mapped high average controllability in both midline parietal and medial prefrontal regions, ${ }^{17}$ suggesting that densely connected "hub" regions strongly influence large-scale functional dynamics. Specific reduction in the same regions in TLE-HS, alongside ipsilateral anterior and lateral temporal decreases, suggests again a selective effect of marked hippocampal neuronal loss on default and temporolimbic networks. The former is one of the most reproducible networks showing activations when participants do not engage in an externally oriented task. Its relevance for restingstate paradigms likely relates to its participation in a broad class of self-generated cognition, including mind-wandering, self-referential thought, and future thinking. ${ }^{51}$ Notably, the mesiotemporal regions have been suggested to closely interact with the default network, representing an important subcomponent of it, ${ }^{20,52}$ possibly reflective of the relevance of mesiotemporal-mediated memory operations for selfgenerated thoughts, ${ }^{53}$ including spatial and semantic memory ${ }^{54}$ but also mental time travel. ${ }^{55}$ Therefore, our control theory simulations suggest that structural connectome rearrangements in patients with HS may compromise the capacity of the mesiotemporal subnetwork in flexibly driving large-scale functional states. Ultimately, these changes may relate to impairments in prospective as well as retrospective memory processes commonly seen in TLE, ${ }^{56-58}$ which are generally more prevalent in patients with marked hippocampal damage than in those with subtle pathology. ${ }^{59,60}$

Both TLE groups presented with bilateral cortical atrophy, in a similar frontocentral and lateral temporal distribution as those shown in our earlier work and a recent multisite metaanalysis. ${ }^{6,24}$ Importantly, despite tendencies for a more extensive pattern in TLE-HS compared to TLE-G, findings did not survive after correction for multiple comparisons, suggesting only little interdependence between white matter connectome reorganization and gray matter structural compromise. Indeed, between-cohort differences in TLE were virtually identical when controlling for cortical thickness. Previous human diffusion MRI data ${ }^{50,61}$ suggest Wallerian degeneration as a potential mediator for the interplay between hippocampal and macroscale connectome anomalies. As hippocampal pathologic grades largely reflect the degree and distribution of cell loss, rather than gliosis, it is plausible that a Wallerian cascade induces proximal and remote limbic deafferentation, which ultimately leads to macroscale network remodeling. Notably, in addition to segmental neuronal loss in the hippocampal formation, $\mathrm{HS}$ is also associated with abnormal migration of granule cells and reorganization of their axons, referred to as granule cell dispersion and mossy fiber sprouting. Pathologic reports have shown that patients with subtle HS show negligible mossy fiber sprouting, as compared to severe sprouting in those with marked neuronal loss. ${ }^{62,63}$ A remodeling of hippocampal internal circuitry may thus contribute to network-level findings at distance, such as those observed in our cohort. Given that the majority of patients included in our study underwent surgery shortly after the presurgical evaluation, we could not longitudinally evaluate white matter networks; such prospective designs may explore whether white matter connectome remodeling shows a progressive course similar to hippocampal and whole-brain gray matter changes in morphology previously reported in TLE. ${ }^{24,64,65}$

Our findings emphasize the importance of taking into consideration the modulatory effects of hippocampal structural alterations when considering TLE from a network perspective. Given recent evidence for network data to provide prognostic markers, particularly in relation to postsurgical outcome, ${ }^{12,66,67}$ it is therefore anticipated that approaches favoring a combination of connectome models, together with advanced structural imaging of the mesiotemporal lobe, will further our understanding of associated cognitive and comorbid psychiatric dysfunction.

\section{Author contributions}

$\mathrm{N}$. Bernasconi and A. Bernasconi recruited patients and acquired the MRI data. B.C. Bernhardt, A. Bernasconi, and N. Bernasconi designed the study. M. Liu, F. Fadaie, B. Caldairou, and B.C. Bernhardt carried out the image processing and network analysis. D.S. Bassett and S. Gu consulted for the network analysis and provided the network control theoretical tools. B.C. Bernhardt wrote the paper and revised it with A. Bernasconi, N. Bernasconi, J. Smallwood, D.S. Bassett, and E. Jefferies.

\section{Study funding}

This work was funded by the Canadian Institutes of Health Research (CIHR MOP-57840 to A.B. and CIHR MOP123520 to N.B.), Natural Sciences and Research Council (NSERC; Discovery-243141 to A.B. and 24779 to N.B.), Epilepsy Canada (Jay \& Aiden Barker Breakthrough Grant in Clinical \& Basic Sciences to A.B.), and Canada First Research Excellence Fund (HBHL-1a-5a-06 to N.B.). F.F. and B.C. received salary support from the Lloyd Carr-Harris Foundation. B.C.B. acknowledges salary support from FRQS and funding from CIHR (FDN-154298) and NSERC (Discovery-1304413), as well as SickKids (NI17-039). D.S.B. acknowledges support from Alfred P. Sloan and John D. and 
Catherine T. MacArthur Foundations, as well as from NIH (NS099348-02).

\section{Disclosure}

The authors report no disclosures relevant to the manuscript. Go to Neurology.org/ $\mathrm{N}$ for full disclosures.

\section{Publication history}

Received by Neurology July 27, 2018. Accepted in final form January 8, 2019.

\section{References}

1. Thom M. Review: hippocampal sclerosis in epilepsy: a neuropathology review. Neuropathol Appl Neurobiol 2014;40:520-543.

2. Briellmann RS, Kalnins RM, Berkovic SF, Jackson GD. Hippocampal pathology in refractory temporal lobe epilepsy: T2-weighted signal change reflects dentate gliosis. Neurology 2002;58:265-271.

3. Cascino GD, Jack CR Jr, Parisi JE, et al. Magnetic resonance imaging-based volume studies in temporal lobe epilepsy: pathological correlations. Ann Neurol 1991;30: 31-36.

4. Goubran M, Bernhardt BC, Cantor-Rivera D, et al. In vivo MRI signatures of hippocampal subfield pathology in intractable epilepsy. Hum Brain Mapp 2016;37: 1103-1119.

5. Kemmotsu N, Girard HM, Bernhardt BC, et al. MRI analysis in temporal lobe epilepsy: cortical thinning and white matter disruptions are related to side of seizure onset. Epilepsia 2011;52:2257-2266.

6. Whelan CD, Altmann A, Botia JA, et al. Structural brain abnormalities in the common epilepsies assessed in a worldwide ENIGMA study. Brain 2018;141:391-408.

7. Campos BM, Coan AC, Beltramini GC, et al. White matter abnormalities associate with type and localization of focal epileptogenic lesions. Epilepsia 2015;56: 125-132.

8. Concha L, Beaulieu C, Gross DW. Bilateral limbic diffusion abnormalities in unilateral temporal lobe epilepsy. Ann Neurol 2005;57:188-196.

9. Focke NK, Yogarajah M, Bonelli SB, Bartlett PA, Symms MR, Duncan JS. Voxel-based diffusion tensor imaging in patients with mesial temporal lobe epilepsy and hippocampal sclerosis. Neuroimage 2008;40:728-737.

10. van Diessen E, Zweiphenning WJ, Jansen FE, Stam CJ, Braun KP, Otte WM. Brain network organization in focal epilepsy: a systematic review and meta-analysis. PLoS One 2014;9:e114606.

11. Liu M, Chen Z, Beaulieu C, Gross DW. Disrupted anatomic white matter network in left mesial temporal lobe epilepsy. Epilepsia 2014;55:674-682.

12. Bonilha L, Helpern JA, Sainju R, et al. Presurgical connectome and postsurgical seizure control in temporal lobe epilepsy. Neurology 2013;81:1704-1710.

13. Bernhardt BC, Bonilha L, Gross DW. Network analysis for a network disorder: the emerging role of graph theory in the study of epilepsy. Epilepsy Behav 2015;50: $162-170$.

14. Richardson MP. Large scale brain models of epilepsy: dynamics meets connectomics. J Neurol Neurosurg Psychiatry 2012;83:1238-1248.

15. Gleichgerrcht E, Kocher M, Bonilha L. Connectomics and graph theory analyses: novel insights into network abnormalities in epilepsy. Epilepsia 2015;56:1660-1668

16. Honey CJ, Sporns O, Cammoun L, et al. Predicting human resting-state functional connectivity from structural connectivity. Proc Natl Acad Sci USA 2009;106: 2035-2040.

17. Gu S, Pasqualetti F, Cieslak M, et al. Controllability of structural brain networks. Nat Commun 2015;6:8414.

18. Wiles L, Gu S, Pasqualetti F, et al. Autaptic connections shift network excitability and bursting. Sci Rep 2017;7:44006.

19. Betzel RF, Gu S, Medaglia JD, Pasqualetti F, Bassett DS. Optimally controlling the human connectome: the role of network topology. Sci Rep 2016;6:30770.

20. Andrews-Hanna JR, Reidler JS, Sepulcre J, Poulin R, Buckner RL. Functionalanatomic fractionation of the brain's default network. Neuron 2010;65:550-562.

21. Blumcke I, Thom M, Aronica E, et al. International consensus classification of hippocampal sclerosis in temporal lobe epilepsy: a Task Force report from the ILAE Commission on Diagnostic Methods. Epilepsia 2013;54:1315-1329.

22. Engel J Jr. Update on surgical treatment of the epilepsies. Neurology 1993;43: 1612-1617.

23. Theodore WH, Kelley K, Toczek MT, Gaillard WD. Epilepsy duration, febrile seizures, and cerebral glucose metabolism. Epilepsia 2004;45:276-279.

24. Bernhardt BC, Bernasconi N, Concha L, Bernasconi A. Cortical thickness analysis in temporal lobe epilepsy: reproducibility and relation to outcome. Neurology 2010;74: 1776-1784.

25. Hong SJ, Bernhardt BC, Caldairou B, et al. Multimodal MRI profiling of focal cortical dysplasia type II. Neurology 2017;88:734-742.

26. Collins DL, Evans AC. Animal: validation and applications of nonlinear registrationbased segmentation. Intern J Pattern Recognit Artif Intell 1997;11:1271-1294.

27. Tzourio-Mazoyer N, Landeau B, Papathanassiou D, et al. Automated anatomical labeling of activations in SPM using a macroscopic anatomical parcellation of the MNI MRI single-subject brain. NeuroImage 2002;15:273-289.
28. Kim JS, Singh V, Lee JK, et al. Automated 3-D extraction and evaluation of the inner and outer cortical surfaces using a Laplacian map and partial volume effect classification. Neuroimage 2005;27:210-221.

29. Liu M, Bernhardt BC, Hong SJ, Caldairou B, Bernasconi A, Bernasconi N. The superficial white matter in temporal lobe epilepsy: a key link between structural and functional network disruptions. Brain 2016;139:2431-2440.

30. Mori S, Crain BJ, Chacko VP, van Zijl PC. Three-dimensional tracking of axonal projections in the brain by magnetic resonance imaging. Ann Neurol 1999;45: 265-269.

31. Hagmann P, Cammoun L, Gigandet X, et al. Mapping the structural core of human cerebral cortex. PLoS Biol 2008;6:e159.

32. Rubinov M, Sporns O. Complex network measures of brain connectivity: uses and interpretations. Neuroimage 2010;52:1059-1069.

33. Latora V, Marchiori M. Efficient behavior of small-world networks. Phys Rev Lett 2001;87:198701.

34. Muldoon SF, Pasqualetti F, Gu S, et al. Stimulation-based control of dynamic brain networks. PLoS Comput Biol 2016;12:e1005076.

35. Liu M, Bernhardt BC, Bernasconi A, Bernasconi N. Gray matter structural compromise is equally distributed in left and right temporal lobe epilepsy. Hum Brain Mapp 2015;37:515-524

36. Kulaga-Yoskovich J, Bernhardt BC, Hong S, et al. Multi-contrast submillimetric 3-Tesla hippocampal subfield segmentation protocol and dataset. Sci Data 2015;2 $1-9$

37. Caldairou B, Bernhardt BC, Kim H, Mansi T, Bernasconi N, Bernasconi A. A surface patch-based segmentation method for hippocampal subfields. Med Image Comput Comput Assist Interv 2016;9901:379-387.

38. Bernhardt BC, Bernasconi A, Liu M, et al. The spectrum of structural and functional imaging abnormalities in temporal lobe epilepsy. Ann Neurol 2016;80: $142-153$.

39. Bernhardt BC, Hong SJ, Bernasconi A, Bernasconi N. Magnetic resonance imaging pattern learning in temporal lobe epilepsy: classification and prognostics. Ann Neurol 2015;77:436-446.

40. Kim H, Bernhardt BC, Kulaga-Yoskovitz J, Caldairou B, Bernasconi A, Bernasconi N. Multivariate hippocampal subfield analysis of local MRI intensity and volume: application to temporal lobe epilepsy. Med Image Comput Comput Assist Interv 2014 17:170-178.

41. Worsley K, Taylor JE, Carbonell F, et al. SurfStat: a Matlab toolbox for the statistical analysis of univariate and multivariate surface and volumetric data using linear mixed effects models and random field theory. Neuroimage 2009;47.

42. Hong S, Bernhardt BC, Schrader DV, Bernasconi N, Bernasconi A. Whole-brain MRI phenotyping of dysplasia-related frontal lobe epilepsy. Neurology 2016;86: 643-650.

43. Benjamini $Y$, Hochberg Y. Controlling the false discovery rate: a practical and powerful approach to multiple testing. J R Stat Soc 1995;57:289-300.

44. Zeisel A, Zuk O, Domany E. FDR control with adaptive procedures and FDR monotonicity. Ann Appl Stat 2011;5:943-968.

45. Hogan RE, Moseley ED, Maccotta L. Hippocampal surface deformation accuracy in T1-weighted volumetric MRI sequences in subjects with epilepsy. J Neuroimaging 2015;25:452-459.

46. Maccotta L, Moseley ED, Benzinger TL, Hogan RE. Beyond the CA1 subfield: local hippocampal shape changes in MRI-negative temporal lobe epilepsy. Epilepsia 2015; 56:780-788.

47. Concha L, Kim H, Bernasconi A, Bernhardt BC, Bernasconi N. Spatial patterns of water diffusion along white matter tracts in temporal lobe epilepsy. Neurology 2012; 79:455-462.

48. Keller SS, Glenn GR, Weber B, et al. Preoperative automated fibre quantification predicts postoperative seizure outcome in temporal lobe epilepsy. Brain 2017;140: $68-82$

49. Bonilha L, Nesland T, Martz GU, et al. Medial temporal lobe epilepsy is associated with neuronal fibre loss and paradoxical increase in structural connectivity of limbic structures. J Neurol Neurosurg Psychiatry 2012;83:903-909.

50. Concha L, Livy DJ, Beaulieu C, Wheatley BM, Gross DW. In vivo diffusion tensor imaging and histopathology of the fimbria-fornix in temporal lobe epilepsy. J Neurosci 2010;30:996-1002.

51. Buckner RL, Andrews-Hanna JR, Schacter DL. The brain's default network: anatomy, function, and relevance to disease. Ann NY Acad Sci 2008;1124:1-38.

52. Vos de Wael R, Lariviere S, Caldairou B, et al. Anatomical and microstructural determinants of hippocampal subfield functional connectome embedding. Proc Natl Acad Sci USA 2018;115:10154-10159.

53. Schooler JW, Smallwood J, Christoff K, Handy TC, Reichle ED, Sayette MA. Metaawareness, perceptual decoupling and the wandering mind. Trends Cogn Sci 2011;15 319-326.

54. Sormaz M, Jefferies E, Bernhardt BC, et al. Knowing what from where: hippocampal connectivity with temporoparietal cortex at rest is linked to individual differences in semantic and topographic memory. Neuroimage 2017;152:400-410.

55. Karapanagiotidis T, Bernhardt BC, Jefferies E, Smallwood J. Tracking thoughts: exploring the neural architecture of mental time travel during mind-wandering. Neuroimage 2017; 147:272-281.

56. Helmstaedter C, Elger CE. Chronic temporal lobe epilepsy: a neurodevelopmental or progressively dementing disease? Brain 2009;132:2822-2830.

57. Tai XY, Bernhardt B, Thom M, et al. Review: neurodegenerative processes in temporal lobe epilepsy with hippocampal sclerosis: clinical, pathological and neuroimaging evidence. Neuropathol Appl Neurobiol 2018;44:70-90. 
58. Adda CC, Castro LH, Alem-Mar e Silva LC, de Manreza ML, Kashiara R. Prospective memory and mesial temporal epilepsy associated with hippocampal sclerosis. Neuropsychologia 2008;46:1954-1964.

59. Bell B, Lin JJ, Seidenberg M, Hermann B. The neurobiology of cognitive disorders in temporal lobe epilepsy. Nat Rev Neurol 2011;7:154-164.

60. Alessio A, Bonilha L, Rorden C, et al. Memory and language impairments and their relationships to hippocampal and perirhinal cortex damage in patients with medial temporal lobe epilepsy. Epilepsy Behav 2006;8:593-600.

61. Beaulieu C. The basis of anisotropic water diffusion in the nervous system: a technical review. NMR Biomed 2002;15:435-455.

62. Proper EA, Jansen GH, van Veelen CW, van Rijen PC, Gispen WH, de Graan PN. A grading system for hippocampal sclerosis based on the degree of hippocampal mossy fiber sprouting. Acta Neuropathol 2001;101:405-409.
63. Schmeiser B, Li J, Brandt A, Zentner J, Doostkam S, Freiman TM. Different mossy fiber sprouting patterns in ILAE hippocampal sclerosis types. Epilepsy Res 2017;136: 115-122.

64. Coan AC, Appenzeller S, Bonilha L, Li LM, Cendes F. Seizure frequency and lateralization affect progression of atrophy in temporal lobe epilepsy. Neurology 2009;73: 834-842.

65. Bernhardt BC, Kim H, Bernasconi N. Patterns of subregional mesiotemporal disease progression in temporal lobe epilepsy. Neurology 2013;81:1840-1847.

66. Gleichgerrcht E, Munsell B, Bhatia S, et al. Deep learning applied to whole-brain connectome to determine seizure control after epilepsy surgery. Epilepsia 2018;59: 1643-1654.

67. Bonilha $\mathrm{L}$, Jensen $\mathrm{JH}, \mathrm{Baker} \mathrm{N}$, et al. The brain connectome as a personalized biomarker of seizure outcomes after temporal lobectomy. Neurology 2015;84:1846-1853. 